STRUCTURAL BIOLOGY COMMUNICATIONS

ISSN 2053-230X

Received 16 January 2018

Accepted 23 March 2018

Edited by J. Agirre, University of York, England

Keywords: O-fucosylation; protein O-fucosyltransferases; epidermal growth factor-like repeats; EGF repeats; thrombospondin type I repeats; TSRs; enzyme mechanisms; GDP-fucose.

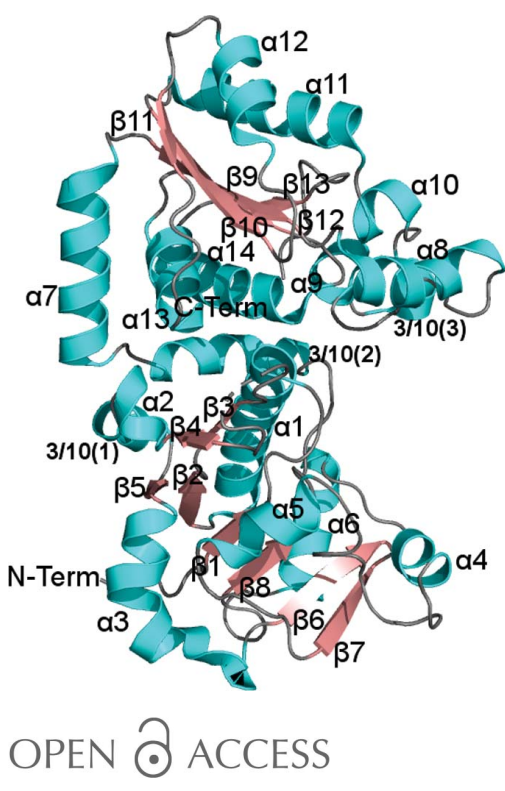

\section{A perspective on structural and mechanistic aspects of protein $O$-fucosylation}

\author{
Erandi Lira-Navarrete $^{\mathrm{a} *}$ and Ramon Hurtado-Guerrero ${ }^{\mathrm{b}, \mathrm{c} *}$ \\ ${ }^{a}$ Copenhagen Center for Glycomics, Department of Cellular and Molecular Medicine, University of Copenhagen, \\ Copenhagen, Denmark, ${ }^{\mathbf{b}} \mathrm{BIFI}$, University of Zaragoza, BIFI-IQFR (CSIC) Joint Unit, Mariano Esquillor s/n, Campus \\ Rio Ebro, Edificio I+D, Zaragoza, Spain, and ${ }^{{ }^{C} F u n d a c i o ́ n ~ A R A I D, ~ A v e n i d a ~ d e ~ R a n i l l a s, ~} 50018$ Zaragoza, Spain. \\ *Correspondence e-mail: erandi@sund.ku.dk, rhurtado@bifi.es
}

Protein $O$-fucosylation is an important post-translational modification (PTM) found in cysteine-rich repeats in proteins. Protein $O$-fucosyltransferases 1 and 2 (PoFUT1 and PoFUT2) are the enzymes responsible for this PTM and selectively glycosylate specific residues in epidermal growth factor-like (EGF) repeats and thrombospondin type I repeats (TSRs), respectively. Within the past six years, crystal structures of both enzymes have been reported, revealing important information on how they recognize protein substrates and achieve catalysis. Here, the structural information available today is summarized and how PoFUT1 and PoFUT2 employ different catalytic mechanisms is discussed.

\section{Introduction}

Fucose is an important biological sugar that can be found as part of various glycoconjugates and is one of the two monosaccharides present in mammals with an L-configuration (Bertozzi \& Rabuka, 2009). Fucose modifications of proteins, mostly known as $O$-fucosylation, play multiple roles in cellular events. In mammals, there are 13 glycosyltransferases (GTs) that are capable of adding a fucose residue using GDP-fucose as the sugar donor, but only two GTs, protein $O$-fucosyltransferases 1 and 2 (PoFUT1 and PoFUT2), can directly glycosylate protein side chains (Schneider et al., 2017). Both PoFUT1 and PoFUT2 transfer fucose from GDP-fucose to serine or threonine residues in cysteine-rich repeats in proteins. However, while PoFUT1 glycosylates epidermal growth factor-like (EGF) repeats within the consensus sequence $\mathrm{C}^{2}-X-X-X-X-\mathrm{S} / \mathrm{T}-\mathrm{C}^{3}$ (Haltom \& Jafar-Nejad, 2015), PoFUT2 glycosylates thrombospondin type I repeats (TSRs) containing Ser/Thr residues located in the consensus sequences $\mathrm{C}^{1}-X-X-\mathrm{S} / \mathrm{T}-\mathrm{C}^{2}$ or $\mathrm{C}^{2}-X-X-\mathrm{S} / \mathrm{T}-\mathrm{C}^{3}$ of TSRs of groups 1 and 2 (see below), respectively (Schneider et al., 2017).

EGF repeats are small domains ranging between 30 and 40 amino acids, characterized by the formation of three disulfide bridges with the arrangement $C^{1}-C^{3}, C^{2}-C^{4}$ and $C^{5}-C^{6}$ (Savage et al., 1973). TSRs are larger than EGF repeats ( $\sim 60$ amino acids) and can be split into two groups owing to their disulfide-bridge arrangement. The disulfide bridges of group 1 TSR are arranged as $C^{1}-C^{5}, C^{2}-C^{6}$ and $C^{3}-C^{4}$, while TSRs of group 2 adopt the pattern $C^{1}-C^{4}, C^{2}-C^{5}$ and $C^{3}-C^{6}$ (LeonhardMelief \& Haltiwanger, 2010). Although the disulfide-bridge arrangement differs between the two groups, the $\mathrm{C}^{2}-\mathrm{C}^{6}$ and $\mathrm{C}^{3}-\mathrm{C}^{6}$ disulfide bridges are conserved (Leonhard-Melief \& Haltiwanger, 2010). PoFUT1 and PoFUT2 both require correctly folded repeats for $O$-fucosylation to take place (Luo, Nita-Lazar et al., 2006; Wang \& Spellman, 1998) and both isoforms are highly selective for each repeat (Luo, Koles et al., 
2006; Luo, Nita-Lazar et al., 2006), suggesting that the different disulfide-bridge arrangements and consensus sequences are essential features for substrate recognition. This review outlines recent progress in unveiling the differences in the reaction mechanisms and protein-substrate recognition of these enzymes, and the importance of $O$-fucosylation in protein-protein interaction.

\section{PoFUT1 and PoFUT2 protein substrates}

The first substrate of PoFUT1 to be identified was the urinary type 1 plasminogen activator (Kentzer et al., 1990). Now, approximately 100 proteins with EGF repeats are predicted to be $O$-fucosylated, although only a few have been confirmed (for a thorough review, see Schneider et al., 2017). The Notch receptors, which are transmembrane type I proteins that form part of the Notch signalling pathway, are the most studied PoFUT1 substrates. Most of the EGF repeats present in the four Notch receptors found in mammals enclose the consensus sequence required for $O$-fucosylation by PoFUT1 (Takeuchi \& Haltiwanger, 2014). Notch ligands, two in Drosophila (Delta and Serrate) and three Delta-like and two Serrate-like ligands in mammals (Dl11, Dll3, Dl14, Jagged1 and Jagged2; D'Souza et al., 2008), also contain EGF repeats that can be $O$-fucosylated by PoFUT1 (Schneider et al., 2017).

Notch glycosylation is an elegant example of how the cell uses protein glycosylation to tune signalling-pathway activity. The elongation of Notch1 $O$-fucose by the addition of an $N$-acetylglucosamine (GlcNAc) moiety by Fringe GTs directs the specificity of the Notch receptors towards Delta and reduces Notch activation by Jagged proteins (Xu et al., 2007). Recent crystallographic complexes between Notch1 and its ligands (DLL4 and Jagged) show that the fucose moiety also plays an essential role in ligand interaction (Luca et al., 2015, 2017). The $O$-fucose moiety at Notch1 EGF12 Thr466 contributes significantly to recognizing DLL4 by hydrogenbonding and hydrophobic interactions with the DLL4 MNNL domain (module at the N-terminus of the Notch ligand; Fig. 1a; Luca et al., 2015). However, the complex between Notch1 and Jagged1 is stabilized by interactions between Notch1 EGF12 Thr466- $O$-fucose and the Jagged1 C2 domain and also between Notch1 EGF8 Thr311- $O$-fucose and Jagged1 EGF3 (Fig. 1b). These interactions between Jagged1 and Notch1 provide an explanation of why Jagged1 binds sixfold more tightly to Notch1 EGFs 8-12 than a construct containing only EGFs 11-12 (Luca et al., 2017). Notch signalling plays a significant role in cell development, and its malfunction can lead to several diseases, including various types of cancer (Allenspach et al., 2002). The glycosylation of Notch receptors may also play a role in aberrant signalling (Takeuchi \& Haltiwanger, 2014), and in this sense it has been reported that PoFUT1 is overexpressed in some cancer types (Kroes et al., 2007; Loo et al., 2013; Yokota et al., 2013).

Despite the prediction of more than 50 protein substrates, very little is known about the TSR $O$-fucose function (Schneider et al., 2017). The first $O$-fucosylated TSRs described in the literature were found on thrombospondin-1
(TSP1; Hofsteenge et al., 2001). Subsequently, PoFUT2 was isolated and characterized, following the realization that PoFUT1 does not glycosylate TSRs (Luo, Nita-Lazar et al., 2006; Luo, Koles et al., 2006). ADAMT and ADAMT-like proteins are a large family of proteins that are predicted to have several TSRs that are potentially $O$-fucosylated by PoFUT2 (Kelwick et al., 2015; Schneider et al., 2017), and $O$-fucose is a requirement for the efficient secretion of some of these proteins (Ricketts et al., 2007; Wang et al., 2007; Vasudevan et al., 2015; Benz et al., 2016). Very recently, it has been reported that depletion of PoFUT2 in Plasmodium falciparum results in attenuated infection of the mosquito vector and human hepatocytes. The authors concluded that this effect was owing to the loss of trafficking of PoFUT2 target proteins (Lopaticki et al., 2017).

\section{The PoFUT1/2 GDP-fucose binding site is highly conserved}

The first reported crystal structures of protein $O$-fucosyltransferases were those of Caenorhabditis elegans PoFUT1 $(\mathrm{CePoFUT1})$ in the unliganded form (PDB entry 3zy4) and in complex with GDP and GDP-fucose (PDB entries $3 z y 3$ and 3zy6) (Lira-Navarrete et al., 2011). The human PoFUT1 (HsPoFUT1) crystal structure was reported in the free form and in the presence of GDP-fucose (PDB entries 5ux6 and 5uxh; Li et al., 2017), and crystal structures of Mus musculus PoFUT1 (MmPoFUT1) in complex with different EGF repeats (PDB entries 5kxh, 5ky0, 5ky2, 5ky3, 5ky4, 5ky5, 5ky7, $5 \mathrm{ky} 8$ and 5ky9) have also been reported (Li et al., 2017). Regarding PoFUT2, there are two crystal structures available for human PoFUT2: in the free form and complexed with GDP-fucose (PDB entries 4ap5 and 4ap6; Chen et al., 2012). In addition, the ternary complex between $\mathrm{CePoFUT2}$, GDP and the human TSR1 (HsTSR1) repeat (PDB entry 5foe) has been described. Note that the latter complex is the only structure of PoFUT2 known to include an acceptor-protein substrate (Valero-González et al., 2016).

Both PoFUT1 and PoFUT2 present the typical GT-B fold, which consists of two Rossmann-like domains facing each other with the active site lying within the resulting deep cleft formed between them (Fig. 2a; Lairson et al., 2008). Although both enzymes share the same type of folding, the superimposition of apo forms of human PoFUT1 and PoFUT2 renders a poor root-mean-square deviation (r.m.s.d.) on 174 equivalent $\mathrm{C}^{\alpha}$ atoms of $3.03 \AA$ (Fig. $2 b$ ), which is in agreement with the observed low sequence identity ( $\sim 28 \%$ identity). This difference between the HsPoFUT1 and HsPoFUT2 crystal structures is mainly attributed to the presence of two prominent loops in HsPoFUT2 that are absent in HsPoFUT1. While loop $_{260-287}$ is located in the C-terminal domain with no apparent function in catalysis, loop $_{141-155}$ contributes to the formation of the cleft in which the acceptor substrate is located (Fig. 2b). HsPoFUT1 also has additional secondary elements that are formed by residues Ser243-Leu284, which encompass three $\alpha$-helices and a $33_{10}$-helix (Fig. 2b). This region prevents the exposure of GDP-fucose to the solvent 
and avoids the binding of a TSR. Similarly, HsPoFUT2 employs loop ${ }_{141-155}$ to selectively bind TSRs in contraposition to EGF repeat acceptor substrates (Valero-González et al., 2016; Li et al., 2017). Superimposition of both human enzymes complexed with GDP-fucose renders an r.m.s.d. on 167 equivalent $\mathrm{C}^{\alpha}$ atoms of $2.62 \AA$, suggesting a higher fold similarity of the enzymes in the presence of the sugar donor in contrast to the free form.

For both enzymes, most of the residues involved in interaction with GDP-fucose are conserved. The essential residues $\operatorname{Arg} 240^{H \text { sPoFUT1 }} / \operatorname{Arg} 294^{H s \text { PoFUT2 }}$ interact with the $\beta$-phosphate of GDP-fucose through hydrogen-bonding and electrostatic

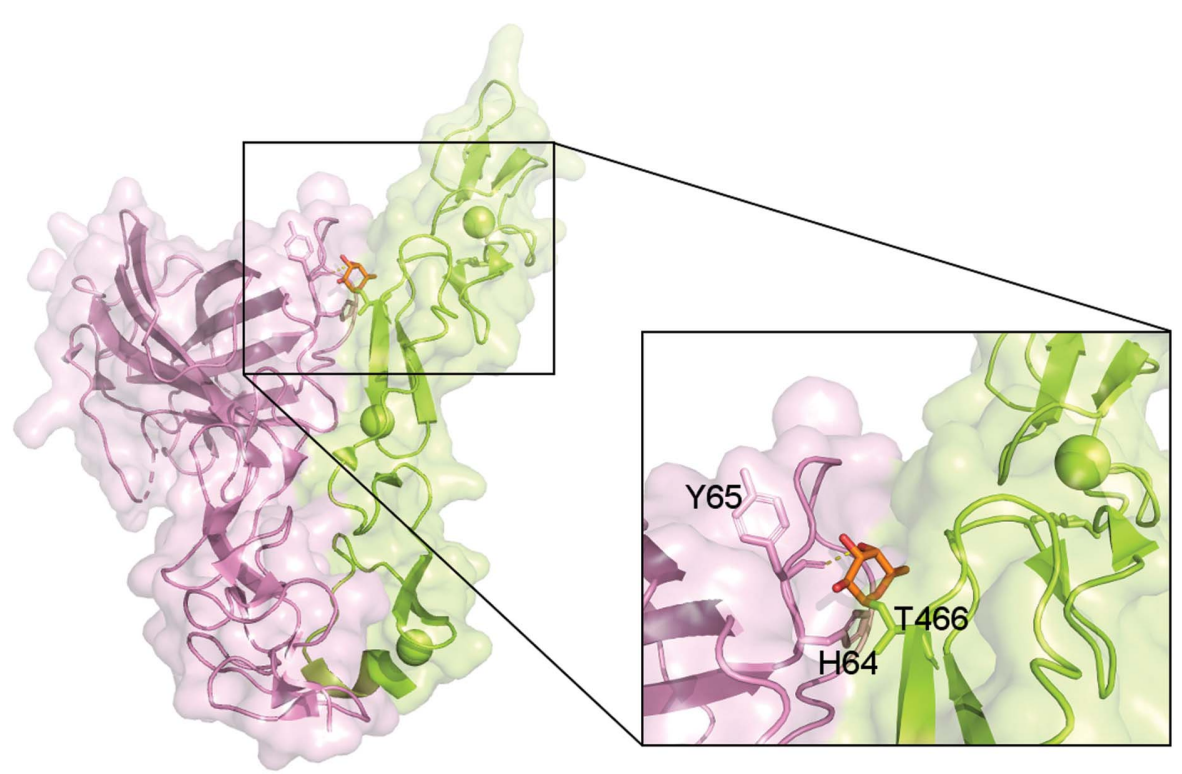

(a)

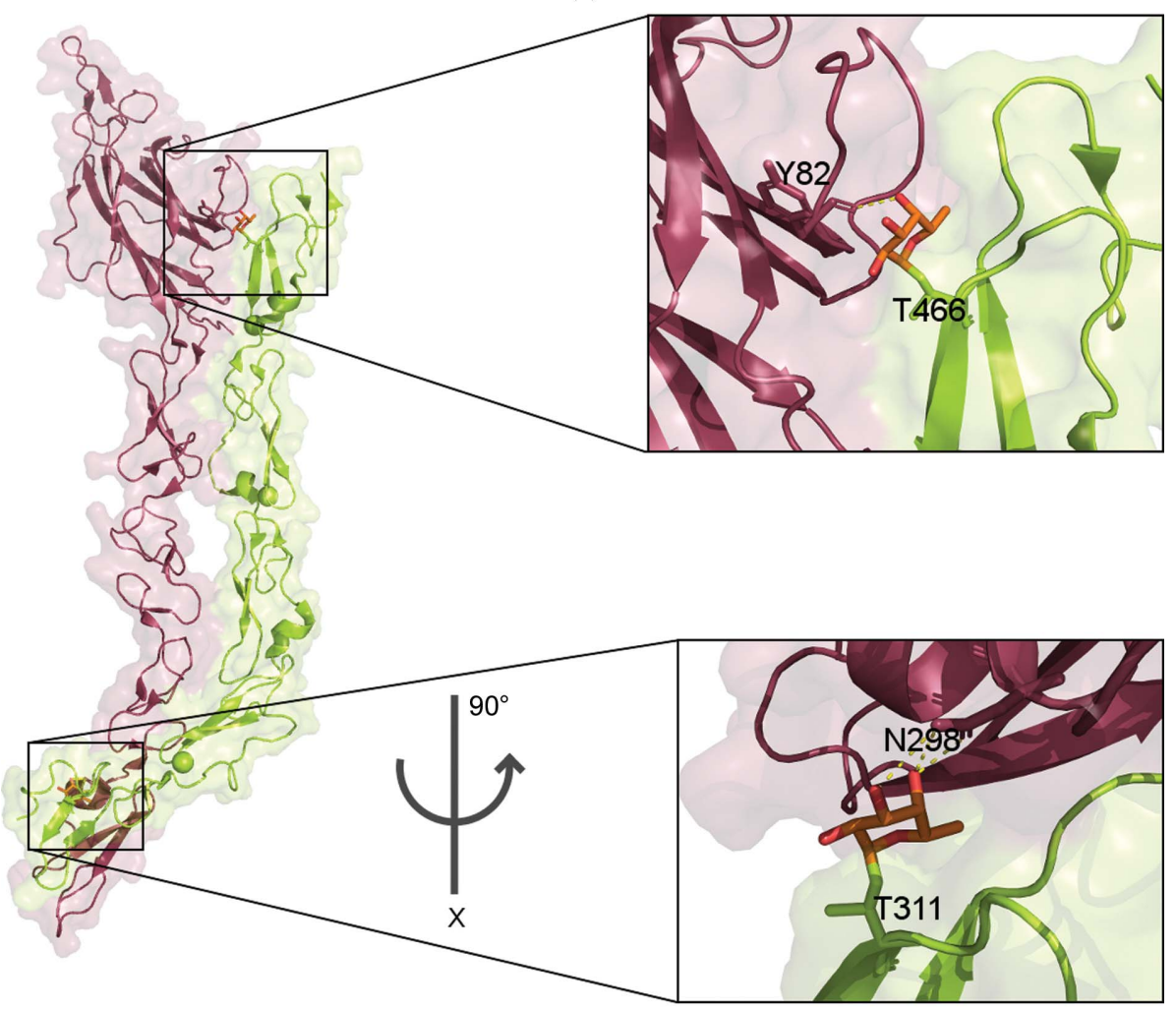

(b)

Figure 1

Importance of Notch fucosylation for ligand interaction. (a) Surface and cartoon representations of the Notch1-DLL4 complex (PDB entry 4xl1; Luca et al., 2015). Notch1 EGFs 11-13 are shown in green and the MNNL-DSL-EGF1 Delta domains are shown in pink. The fucose (orange) modification of Notch1 Thr466 and its interactions with DLL4 residues are depicted in the left panel. (b) Surface and cartoon representation of the Notch1-Jagged1 complex (PDB entry 5uk5; Luca et al., 2017). Notch 1 EGFs 8-12 are shown in green, the C2-DSL-EGF1-3 Jagged domains are shown in red and Notch fucose is shown in orange. The interactions of the Notch1 fucose modifications of Thr466 and Thr311 are depicted in the upper and lower panels, respectively. $\mathrm{Ca}^{2+}$ ions are shown as green spheres. 
interactions, which are conserved in other fucosyltransferases (Martinez-Duncker et al., 2003; Okajima et al., 2005; LiraNavarrete et al., 2011; Chen et al., 2012; McMillan et al., 2017). The GDP moiety is tethered by additional interactions with Asn46/Asn57, His238/His292, Asp340/Asp371, Ser356/Ser387, Ser357/Thr388 and Phe358/Phe389 of HsPoFUT1 and HsPoFUT2, respectively (Fig. 3). GDP also interacts with the backbones of Phe 44 and Gly45 in HsPoFUT1. Contrary to the high level of conservation between the interacting residues of $H s$ PoFUT1 and HsPoFUT2 with the GDP moiety, the residues recognizing the fucose moiety are not conserved. In particular, the fucose moiety is stabilized by interactions with Arg43/ Asp244 of $H s$ PoFUT1 and Pro53/Gly55 of HsPoFUT2 (Fig. 3).

\section{PoFUT1 and PoFUT2 deploy different strategies for protein-substrate recognition}

The structures of both enzymes in complex with acceptor substrates highlight significant differences. Complexes between MmPoFUT1 and four different EGF repeats reveal that the binding mode of all repeats is preserved $(\mathrm{Li}$ et al., 2017). The EGF repeats locate near a hairpin formed by amino acids Val72-Ser91, which are highly conserved among different species (Lira-Navarrete et al., 2011). This particular hairpin moves to maintain contact with the EGF $C^{5}-C^{6}$ subdomain through hydrophobic interactions with residues His80 or Phe85 (Li et al., 2017). Other conserved interactions are made by MmPoFUT1 residues Phe266 and Met267 and an apolar residue located next to the fourth cysteine in the EGF repeats (Fig. $4 a$; Li et al., 2017). Finally, primary interactions between MmPoFUT1 and the different EGF repeats are formed by amino acids from the inner part of the MmPoFUT1 groove and the EGF consensus sequence. Within the consensus sequence, the hydrogen-bond interaction between the acceptor Ser/Thr and Asn51 $1^{\text {MmPoFUT1 }}$ is of the utmost importance for catalytic purposes ( $\mathrm{Li}$ et al., 2017).

Looking at the CePoFUT2-GDP-HsTSR1 ternary complex, it is evident that both the interactions between the
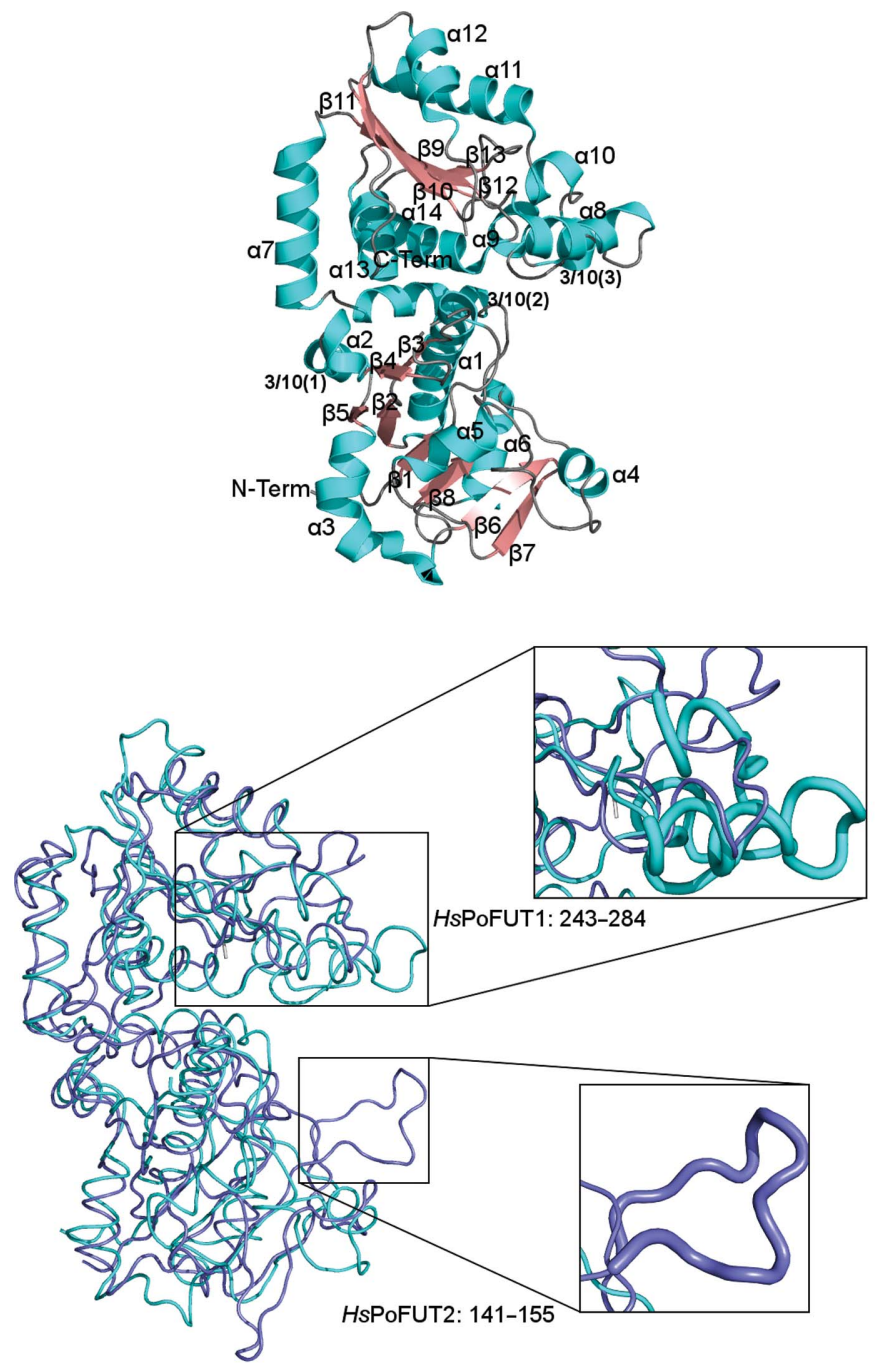

Figure 2

(a) Cartoon representations of the human PoFUT1 (PDB entry 5ux6; McMillan et al., 2017) and PoFUT2 (PDB entry 4ap5; Chen et al., 2012) structures. Secondary structures are shown for HsPoFUT1 with helices in cyan and $\beta$-sheets in salmon; for $H s$ PoFUT2 helices are shown in slate blue and $\beta$-sheets in magenta. (b) Superimposed structures of HsPoFUT1 (cyan) and HsPoFUT2 (slate blue). Main secondary-structure differences are highlighted in boxes. 
CePoFUT2-HsTSR1 and MmPoFUT1-EGF complexes and the arrangement of the TSR and EGF repeats differ (ValeroGonzález et al., 2016; Li et al., 2017). The CePoFUT2-HsTSR1 complex is partly supported by direct interactions between two hydrophobic patches of $\mathrm{CePoFUT2}$ and nonconserved residues of $H s$ TSR1 (Fig. $4 b$ ). Three of the ten direct interactions between $C e$ PoFUT2 and HsTSR1 are conserved for other TSRs, suggesting that the complex is stabilized by a limited number of direct interactions. Within these three interactions, the hydrogen bond between the acceptor Ser or Thr and the catalytic base Glu52 is essential for catalysis (Valero-González et al., 2016).

A striking difference between the MmPoFUT1-EGF and CePoFUT2-HsTSR1 complexes is the large number of water molecules that are present in the interface of the latter. These water molecules mediate an important number of interactions through hydrogen bonds between the enzyme and HsTSR1 (Fig. 4b; Valero-González et al., 2016). The interactions provide an explanation at the molecular level of how PoFUT2 recognizes multiple dissimilar TSRs (Leonhard-Melief \& Haltiwanger, 2010; Kakuda \& Haltiwanger, 2014). Therefore, both PoFUT1 and PoFUT2 deploy different strategies to identify their acceptor-protein substrates. PoFUT2 recognizes TSRs by using a limited number of direct conserved interactions complemented by a large number of hydrogen-bond interactions mediated by water molecules (Valero-González et al., 2016). Meanwhile, PoFUT1 uses a water-filled cavity to accommodate the EGF loop $\mathrm{C}^{1}-\mathrm{C}^{2}$ (Li et al., 2017), although the main interactions rely on conserved direct hydrogen bonds between the enzyme and its protein substrate.

\section{Catalytic mechanisms of PoFUT1 and PoFUT2}

The ternary complexes also provided a better understanding of how the two enzymes achieve catalysis. These proteins are inverting GTs, implying that the acceptor Ser/Thr makes a nucleophilic attack on the nucleotide sugar anomeric $\mathrm{C}$ atom from the opposite side to the leaving nucleotide. As a result, this action inverts the anomeric stereochemistry (Lairson et al., 2008). In this mechanism, deprotonation of the acceptor hydroxyl group by a catalytic base, usually an aspartate or a glutamate, is required to increase the nucleophilic character of the acceptor residue and is a prior step to the attack of the acceptor on the anomeric $\mathrm{C}$ atom (Lairson et al., 2008; Breton et al., 2012). While an amino acid acting as a catalytic base is present in PoFUT2 (Glu54/Glu52 in human PoFUT2 and $\mathrm{CePoFUT2}$, respectively), an equivalent residue is not found in PoFUT1. As expected, mutating Glu54 and Arg294 in HsPoFUT2 revealed that these two amino acids are essential for catalytic activity (Chen et al., 2012). Furthermore, the CePoFUT2-GDP-HsTSR1 complex, together with molecular dynamics, supports the role of a glutamate as the catalytic base. In this crystal structure, Glu52 was engaged in a hydrogen bond to the acceptor serine in the HsTSR1 repeat (Valero-González et al., 2016). Overall, these data support an $\mathrm{S}_{\mathrm{N}}$ 2-like mechanism for PoFUT2 (Fig. 5b), which is the typical mechanism reported for most inverting glycosyltransferases (Lairson et al., 2008).

In contrast, an $\mathrm{S}_{\mathrm{N}} 1$-like mechanism was proposed for PoFUT1 (Fig. 5a) in which Asn 43 of CePoFUT1 positions the incoming acceptor and Arg240 facilitates the cleavage of the glycosidic bond by interacting with the $\beta$-phosphate group (Lira-Navarrete et al., 2011). Michaelis complexes of MmPoFUT1 also support an $\mathrm{S}_{\mathrm{N}} 1$-like mechanism and confirm a similar arrangement as above. The equivalent residues in MmPoFUT1, Asn51 and Arg245, are engaged in hydrogen bonds to the acceptor residue and the $\beta$-phosphate group, respectively. Arg240 ${ }^{\text {CePoFUT1 }} / \operatorname{Arg} 245^{\text {MmPoFUT1 }}$ could also favour the reaction by stabilizing GDP. In addition, the acceptor hydroxyl group is close to a water molecule that is engaged in a hydrogen bond to the $\beta$-phosphate $\mathrm{O}$ atom. In concordance with an $\mathrm{S}_{\mathrm{N}} 1$-like mechanism, this water molecule

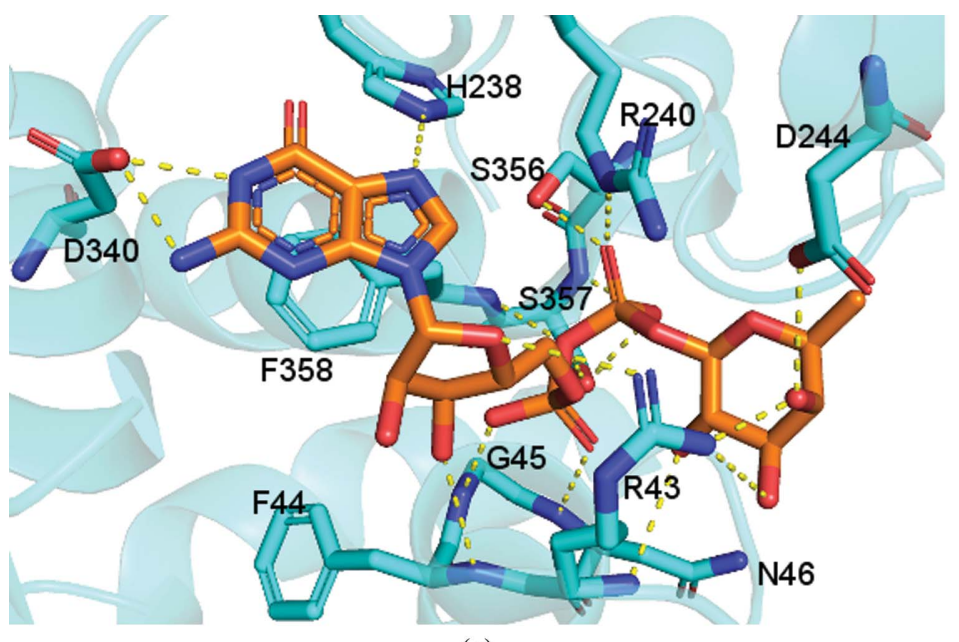

(a)

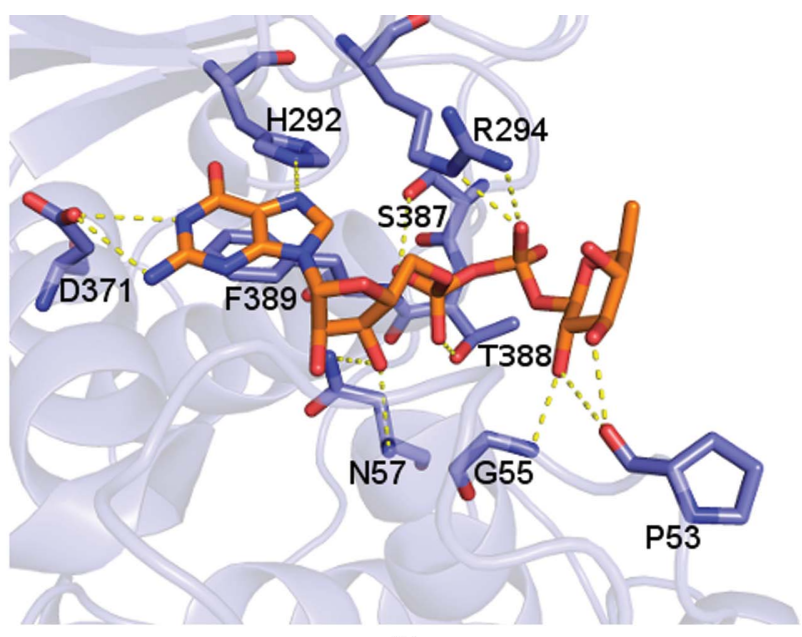

(b)

Figure 3

Comparison of the GDP-fucose binding site in (a) HsPoFUT1 (PDB entry 5uxh; McMillan et al., 2017) and (b) HsPoFUT2 (PDB entry 4ap6; Chen et al., 2012). GDP-fucose is shown in stick representation with orange $\mathrm{C}$ atoms. Amino acids of $H s$ PoFUT1 and $H s$ PoFUT2 that interact with GDP-fucose are represented as sticks with cyan and slate blue $\mathrm{C}$ atoms, respectively. 
could promote catalysis by providing a proton relay serving to shuttle the acceptor hydroxyl proton to the GDP $\beta$-phosphate
$\mathrm{O}$ atom, which acts as the catalytic base (Li et al., 2017). This reaction mechanism of PoFUT1 differs from that proposed for

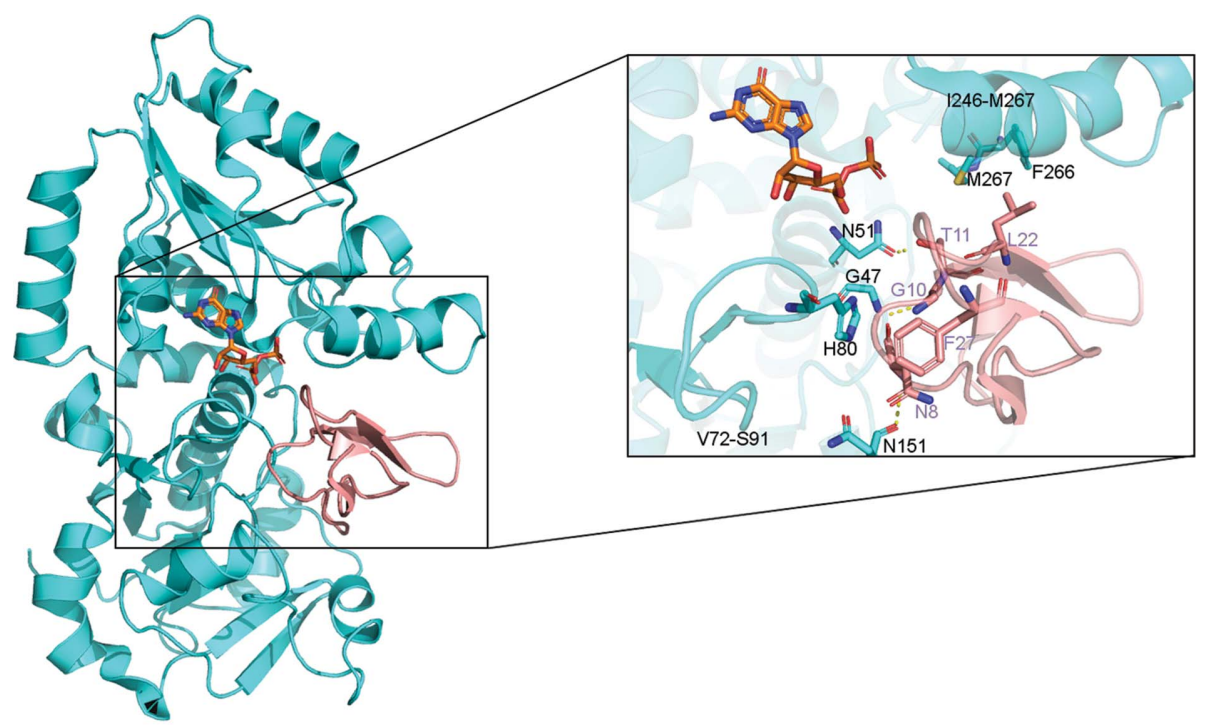

(a)

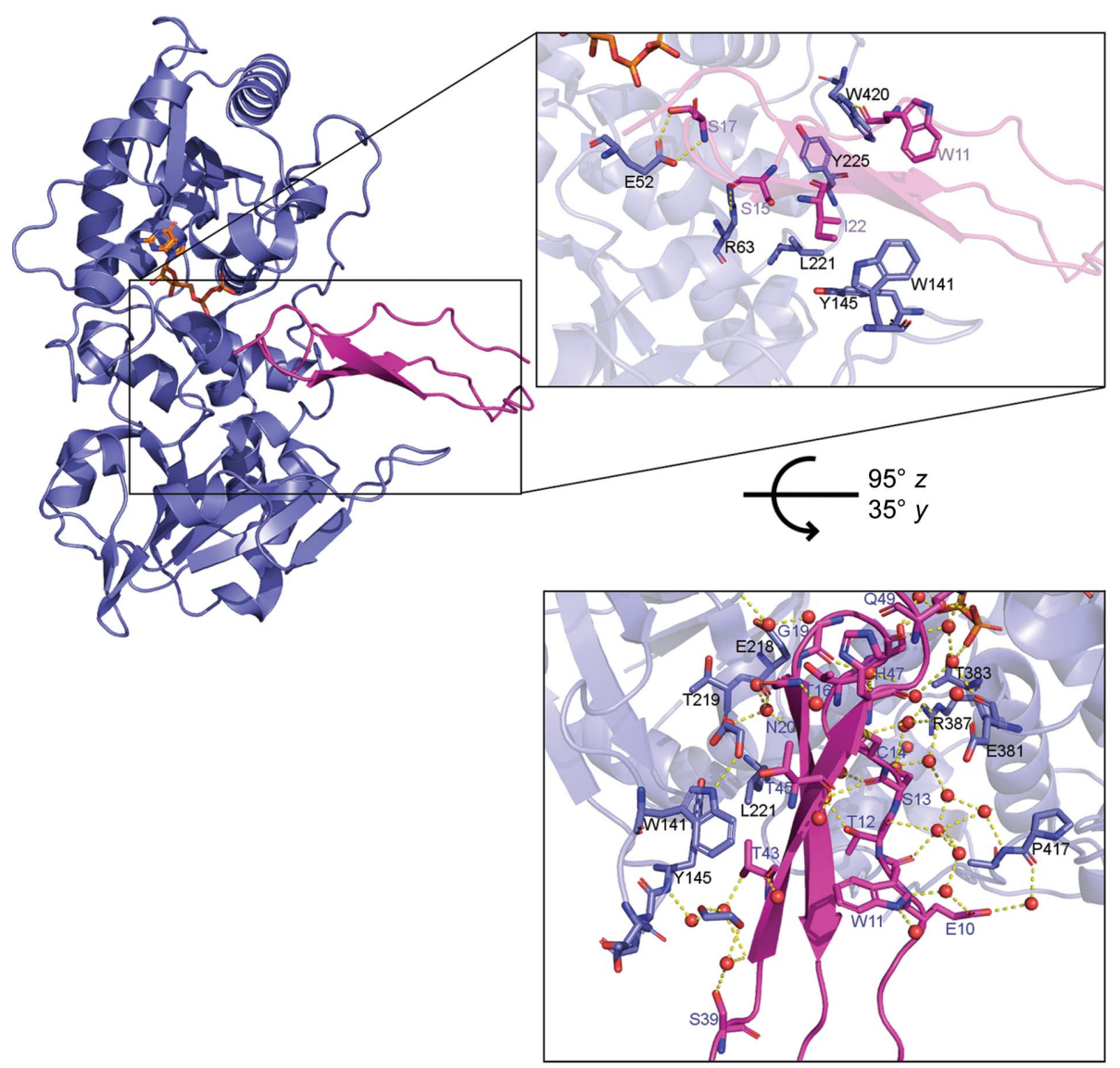

(b)

Figure 4

Comparison of the PoFUT1 and PoFUT2 acceptor-binding sites. (a) Left: cartoon representation of MmPoFUT1 (cyan) in complex with MmEGF26 (salmon) and GDP (depicted as sticks with orange C atoms; PDB entry 5ky4; Li et al., 2017). Right: close-up view of the EFG binding site. The interacting amino acids of MmPoFUT1 and MmEGF26 are shown as sticks with cyan and salmon C atoms, respectively. (b) Left: cartoon representation of CePoFUT2 (slate blue) in complex with HsTSR1 (magenta) and GDP (sticks with orange C atoms; PDB entry 5foe; Valero-González et al., 2016). Upper right box: close-up view of the TSR binding site. C atoms of interacting residues are shown as sticks in slate blue (CePoFUT2) and magenta (HsTSR1). Lower right box: close-up view of the TSR binding site rotated $95^{\circ}$ around the $z$ axis and $35^{\circ}$ around the $y$ axis. Amino-acid colours are the same as in the upper panel and water molecules are shown as red spheres. 

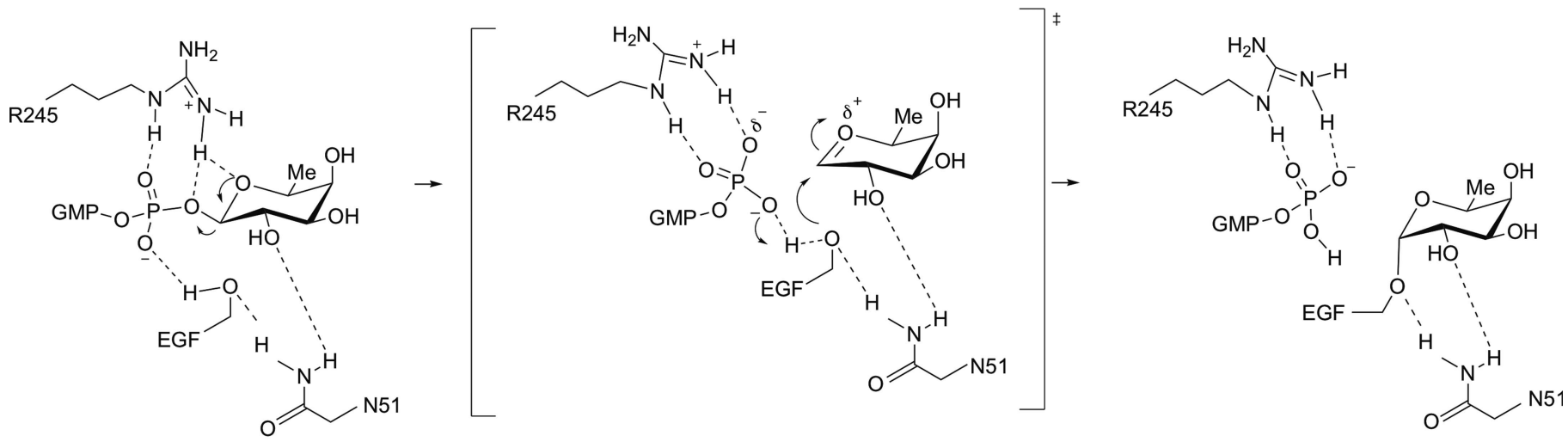

(a)

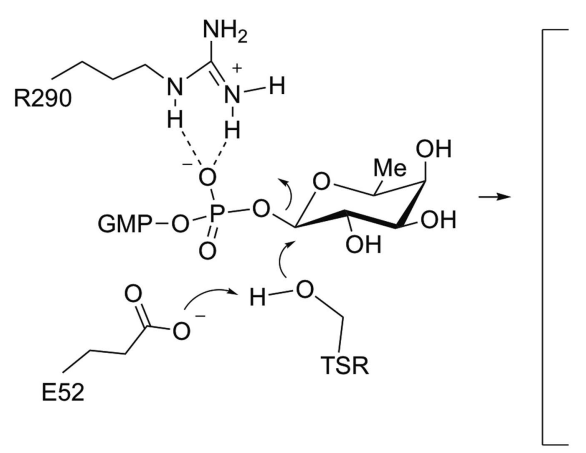

Figure 5

Catalytic mechanisms of PoFUT1 and PoFUT2. (a) The $\mathrm{S}_{\mathrm{N}} 1$-like catalytic mechanism proposed for PoFUT1. Note that a water-mediated proton transfer might also promote the above mechanism. (b) The $\mathrm{S}_{\mathrm{N}} 2$-like mechanism proposed for PoFUT2.

PoFUT2, and consists of prior cleavage of the glycosidic bond, followed by attack of the pre-activated acceptor hydroxyl group on the anomeric $\mathrm{C}$ atom.

\section{Final remarks}

PoFUT1 and PoFUT2 are glycosyltransferases that share great resemblances, including catalysis of the same type of PTM, architecture and the recognition of proteins with cysteine-rich repeats (Chen et al., 2012; Schneider et al., 2017). However, significant differences are present at the primary-structure and secondary-structure levels, accounting for the different arrangements of EGF repeats and TSRs in the binding site, the recognition mode of EGF repeats and TSRs, and the reaction mechanism. These differences explain why these enzymes serve specific substrates and are not capable of crossrecognizing their acceptor substrates. PoFUT2 has evolved to glycosylate two different groups of TSRs (Kakuda \& Haltiwanger, 2014). In doing so, PoFUT2 employs a different strategy to recognize its TSRs by using a water-molecule network that mediates enzyme-protein substrate interactions (Valero-González et al., 2016). In addition, while the $\mathrm{S}_{\mathrm{N}} 2$-like mechanism of PoFUT2 is well accepted for inverting GTs, the atypical $\mathrm{S}_{\mathrm{N}}$ 1-like mechanism proposed for PoFUT1 needs further validation by additional experiments.

We expect that these findings will be able to be leveraged for the development of inhibitors/modulators of PoFUT1/2 that would be useful for providing further insights into the role of this PTM in animal models and for diseases and pathologies associated with these enzymes.

\section{Funding information}

The work of the authors was supported by a postdoctoral EMBO fellowship ALTF 1553-2015 to EL-N co-funded by the European Commission (LTFCOFUND2013, GA-2013609409) and Marie Curie Action, the ARAID Foundation, MICINN (CTQ2013-44367-C2-2-P and BFU2016-75633-P) and DGA (B89).

\section{References}

Allenspach, E. J., Maillard, I., Aster, J. C. \& Pear, W. S. (2002). Cancer Biol. Ther. 1, 466-476.

Benz, B. A., Nandadasa, S., Takeuchi, M., Grady, R. C., Takeuchi, H., LoPilato, R. K., Kakuda, S., Somerville, R. P. T., Apte, S. S., Haltiwanger, R. S. \& Holdener, B. C. (2016). Dev. Biol. 416, 111122.

Bertozzi, C. R. \& Rabuka, D. (2009). Essentials of Glycobiology, 2nd ed., edited by A. Varki, R. D. Cummings, J. D. Esko, H. H. Freeze, P. Stanley, C. R. Bertozzi, G. W. Hart \& M. E. Etzler, pp. 23-36. New York: Cold Spring Harbor Laboratory Press.

Breton, C., Fournel-Gigleux, S. \& Palcic, M. M. (2012). Curr. Opin. Struct. Biol. 22, 540-549.

Chen, C.-I., Keusch, J. J., Klein, D., Hess, D., Hofsteenge, J. \& Gut, H. (2012). EMBO J. 31, 3183-3197. 
D'Souza, B., Miyamoto, A. \& Weinmaster, G. (2008). Oncogene, 27, 5148-5167.

Haltom, A. R. \& Jafar-Nejad, H. (2015). Glycobiology, 25, 1027-1042.

Hofsteenge, J., Huwiler, K. G., Macek, B., Hess, D., Lawler, J., Mosher, D. F. \& Peter-Katalinic, J. (2001). J. Biol. Chem. 276, 64856498.

Kakuda, S. \& Haltiwanger, R. S. (2014). Handbook of Glycosyltransferases and Related Genes, 2nd ed., edited by N. Taniguchi, K. Honke, M. Fukuda, H. Narimatsu, Y. Yamaguchi \& T. Angata, pp. 623-633. Tokyo: Springer.

Kelwick, R., Desanlis, I., Wheeler, G. N. \& Edwards, D. R. (2015). Genome Biol. 16, 113.

Kentzer, E. J., Buko, A., Menon, G. \& Sarin, V. K. (1990). Biochem. Biophys. Res. Commun. 171, 401-406.

Kroes, R. A., Dawson, G. \& Moskal, J. R. (2007). J. Neurochem. 103, Suppl. 1, 14-24.

Lairson, L. L., Henrissat, B., Davies, G. J. \& Withers, S. G. (2008). Annu. Rev. Biochem. 77, 521-555.

Leonhard-Melief, C. \& Haltiwanger, R. S. (2010). Methods Enzymol. 480, 401-416.

Li, Z., Han, K., Pak, J. E., Satkunarajah, M., Zhou, D. \& Rini, J. M. (2017). Nature Chem. Biol. 13, 757-763.

Lira-Navarrete, E., Valero-González, J., Villanueva, R., MartínezJúlvez, M., Tejero, T., Merino, P., Panjikar, S. \& Hurtado-Guerrero, R. (2011). PLoS One, 6, e25365.

Loo, L. W. M., Tiirikainen, M., Cheng, I., Lum-Jones, A., Seifried, A., Church, J. M., Gryfe, R., Weisenberger, D. J., Lindor, N. M., Gallinger, S., Haile, R. W., Duggan, D. J., Thibodeau, S. N., Casey, G. \& Le Marchand, L. (2013). Genes Chromosomes Cancer, 52, 450-466.

Lopaticki, S., Yang, A. S. P., John, A., Scott, N. E., Lingford, J. P., O'Neill, M. T., Erickson, S. M., McKenzie, N. C., Jennison, C., Whitehead, L. W., Douglas, D. N., Kneteman, N. M., GoddardBorger, E. D. \& Boddey, J. A. (2017). Nature Commun. 8, 561.

Luca, V. C., Jude, K. M., Pierce, N. W., Nachury, M. V., Fischer, S. \& Garcia, K. C. (2015). Science, 347, 847-853.

Luca, V. C., Kim, B. C., Ge, C., Kakuda, S., Wu, D., Roein-Peikar, M., Haltiwanger, R. S., Zhu, C., Ha, T. \& Garcia, K. C. (2017). Science,
355, 1320-1324.

Luo, Y., Koles, K., Vorndam, W., Haltiwanger, R. S. \& Panin, V. M. (2006). J. Biol. Chem. 281, 9393-9399.

Luo, Y., Nita-Lazar, A. \& Haltiwanger, R. S. (2006). J. Biol. Chem. 281, 9385-9392.

Martinez-Duncker, I., Mollicone, R., Candelier, J.-J., Breton, C. \& Oriol, R. (2003). Glycobiology, 13, 1C-5C.

McMillan, B. J., Zimmerman, B., Egan, E. D., Lofgren, M., Xu, X., Hesser, A. \& Blacklow, S. C. (2017). Glycobiology, 27, 777786.

Okajima, T., Xu, A., Lei, L. \& Irvine, K. D. (2005). Science, 307, 1599 1603.

Ricketts, L. M., Dlugosz, M., Luther, K. B., Haltiwanger, R. S. \& Majerus, E. M. (2007). J. Biol. Chem. 282, 17014-17023.

Savage, C. R. Jr, Hash, J. H. \& Cohen, S. (1973). J. Biol. Chem. 248, 7669-7672.

Schneider, M., Al-Shareffi, E. \& Haltiwanger, R. S. (2017). Glycobiology, 27, 601-618.

Takeuchi, H. \& Haltiwanger, R. S. (2014). Biochem. Biophys. Res. Commun. 453, 235-242.

Valero-González, J., Leonhard-Melief, C., Lira-Navarrete, E., Jiménez-Osés, G., Hernández-Ruiz, C., Pallarés, M. C., Yruela, I., Vasudevan, D., Lostao, A., Corzana, F., Takeuchi, H., Haltiwanger, R. S. \& Hurtado-Guerrero, R. (2016). Nature Chem. Biol. 12, 240 246.

Vasudevan, D., Takeuchi, H., Johar, S. S., Majerus, E. \& Haltiwanger, R. S. (2015). Curr. Biol. 25, 286-295.

Wang, L. W., Dlugosz, M., Somerville, R. P. T., Raed, M., Haltiwanger, R. S. \& Apte, S. S. (2007). J. Biol. Chem. 282, 17024-17031.

Wang. Y. \& Spellman, M. W. (1998). J. Biol. Chem. 273, 81128118.

Xu, A., Haines, N., Dlugosz, M., Rana, N. A., Takeuchi, H., Haltiwanger, R. S. \& Irvine, K. D. (2007). J. Biol. Chem. 282, 35153-35162.

Yokota, S., Ogawara, K., Kimura, R., Shimizu, F., Baba, T., Minakawa, Y., Higo, M., Kasamatsu, A., Endo-Sakamoto, Y., Shiiba, M., Tanzawa, H. \& Uzawa, K. (2013). Int. J. Oncol. 43, $1864-1870$ 